\section{Visión Electrónica Más que un estado sólido \\ https://doi.org/10.14483/issn.2248-4728}

UNIVERSIDAD DISTRITAL

FRANCISCO JOSÉ DE CALDAS

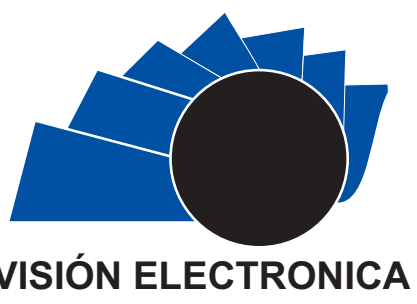

Visión investigadora

\title{
Comportamiento de rangos articulares con alineación en amputados transtibiales
}

\author{
Behavior of articular ranges with alignment of transtibial amputees
}

Lely Adriana Luengas Contreras ${ }^{1}$, Javier López Macías ${ }^{2}$, Giovanni Sánchez Prieto ${ }^{3}$

\section{INFORMACIÓN DEL ARTICULO}

Historia del articulo

Enviado: $15 / 11 / 2017$

Recibido: 08/03/2018

Aceptado: 29/05/2018

\section{Palabras clave:}

Alineación de prótesis transtibiales, Alineación estática, Análisis estadístico.

\section{Keywords:}

Alignment of transtibial

prostheses

Static alignment

Statistical analysis.

\section{RESUMEN}

La falta de información objetiva relacionada con la alineación de las prótesis es un fenómeno común en el área. Cuando aparece una ubicación angular inadecuada de la cavidad protésica transtibial, se ven afectados los parámetros biomecánicos en la articulación de los amputados; también afecta la comodidad del amputado en la posición de pie. La presente investigación da cuenta de los efectos de la desalineación de la prótesis transtibial en los parámetros biomecánicos al medirse en términos de ángulos de las extremidades inferiores, cuando el sujeto se encuentra en la posición bipedestada estática. Se midieron siete condiciones de alineación en cada uno de los 6 participantes del estudio, mientras que al participante siete (7) solo se le localizaron seis condiciones, para un total de 144 pruebas. Un sistema de medición de ángulos (goniómetros digitales) permitió detectar y almacenar los cambios de los rangos articulares. El análisis estadístico de las mediciones realizadas mostró una variación intra y entre sujetos, por lo que se estableció una metodología para la obtención del modelo de alineación estática.

\section{ABSTRACT:}

The lack of objective information concerned to prostheses alignment is a common phenomenon in the area. When an inadequate angular location of the transtibial prosthetic socket appears, the biomechanical parameters in the joint in the amputees are affected; it also affects the amputee's comfort in the standing position. The effects of transtibial prosthesis malalignment on biomechanical parameters were measured in terms of lower limb angles, when the subject is in the static standing position. Seven insertion alignment conditions were measured in each of the 6 participants of the study, whereas participant seven (7) only six conditions were located, for a total number of 144 tests. The socket angle and the joint angles, were recorded in computer files. The statistical analysis of the measurements carried out showed variation intra and inter subjects, so a methodology for the obtaining of the static alignment model was stated.

\footnotetext{
1 Ingeniera Electrónica, Universidad Autónoma de Colombia, Colombia. Esp. En Pedagogía y Docencia Universitaria, Universidad de San Buenaventura, Colombia. MSc. En Ingeniería Eléctrica, Universidad de los Andes, Colombia. Ph.D. En Ingeniería, Pontificia Universidad Javeriana, Colombia. Docente Universidad Distrital Francisco José de Caldas, Colombia. Correo Electrónico: laluengasc@,udistrital.edu.co._ORCID: https://orcid.org/o000-0002-3600$\underline{4666 .}$

2 Ingeniero de Sistemas. Esp. En Ingeniería de software. MSc.(c) En Informática Educativa. Investigador en el grupo Robótica Móvil Autónoma "ROMA”. Docente Universidad Distrital Francisco José de Caldas, Colombia. Correo Electrónico: jlopezmacias@udistrital.edu.co. ORCID: https://orcid.org/o0o00003-4562-9454.

3 Ingeniero Electrónico, Universidad Autónoma de Colombia, Colombia. Esp. En Pedagogía y Docencia Universitaria, Universidad de San Buenaventura, Colombia. Docente Universidad San Buenaventura, Colombia. Correo Electrónico: ing.dprtecnologias@usbbog.edu.co. ORCID: https://orcid.org/o0o00003-2108-6491.

Citar este artículo como: L. A. Luengas-Contreras, J. López-Macías y G. Sánchez-Prieto, “Comportamiento de rangos articulares con alineación en amputados transtibiales", Visión electrónica, algo más que un estado sólido, vol. 1, no. 1, Edición especial, enero-junio 2018.DOI revista: https://doi.org/10.14483/issn.22484728.

Visión Electrónica: Algo más que un estado sólido ISSN 1909-9746 E-ISSN 2248-4728 Volumen 1 número 1- Edición Especial Enero -Junio de 2018
} 


\section{Introducción}

Actualmente en el mundo 1,5 de cada 1.000 habitantes son amputados, es decir: alrededor de 10 millones de personas [1]. En Colombia, el Departamento Nacional de Estadística (DANE, por sus siglas) censó en 2005, aproximadamente 770 mil personas que presentaban dificultades en el movimiento del cuerpo y los miembros inferiores para realizar actividades tales como desplazarse de un sitio a otro, lo que conlleva a tener limitaciones para caminar [2]. Dentro del grupo censado se encontraban víctimas por minas antipersonales, que entre 1990 y noviembre de 2014 han ascendido a cerca de 8.791 heridos -algunos de ellos han sufrido amputación de extremidades inferiores [3], siendo la amputación transtibial la más común, donde existe una pérdida de una o ambas piernas debajo de la articulación de la rodilla.

De lo reportado, uno de los elementos usados en la rehabilitación de un amputado es la prótesis, cuya función es reemplazar las estructuras anatómicas perdidas. Al colocar una prótesis en un segmento corporal se requiere acondicionar el ajuste entre las superficies conectadas, si la prótesis es transtibial se realiza alineamiento femorotibial, el cual se puede definir como "la posición relativa del encaje con respecto al eje de la rodilla, la pierna y el pie... y sirve para equilibrar las fuerzas y los momentos que actúan sobre las articulaciones y los segmentos residuales del miembro inferior", otra forma de expresarlo es "por el ángulo formado por los ejes anatómicos o mecánicos de dichos segmentos óseos" [4], [5].

En consecuencia, se ha reconocido por mucho tiempo que la alineación de prótesis tiene una influencia sustancial en la calidad de vida de los usuarios, pues como requisito previo para las actividades diarias con su prótesis el amputado transtibial debe ser capaz de soportarla cómodamente, no presentar dolores, inestabilidad, entre otros $[6],[7]$.

De acuerdo a lo expuesto, se evidencia que la alineación estática es el primer contacto del paciente con la prótesis; el protesista revisa la conexión entre el muñón residual y el encaje, el nivel de la pelvis, la estabilidad y la longitud ipsilateral y contralateral de los miembros inferiores: todo esto cuando el paciente se encuentra bipedestado [8], [9].

Por lo anterior, y teniendo en cuenta la necesidad de tener información relevante sobre el comportamiento de parámetros biomecánicos acorde con la alineación estática, se propuso y ejecutó la presente investigación, cuyo propósito general es la caracterización de rangos articulares en la alineación estática de prótesis transtibiales.

La investigación desarrollada presentó dos etapas, la primera fue la creación de una base de datos de la afectación de la posición angular del encaje en el plano sagital sobre rangos articulares del amputado; en la segunda etapa se exploraron los datos medidos y se obtuvieron características de comportamiento.

A continuación se presenta la estructura planteada para este documento: La sección 2 aborda la metodología propuesta y desarrollada para alcanzar los objetivos propuestos y la validación de las hipótesis trazadas. En la sección 3 están los resultados, allí se incluye el análisis de datos. La sección 4 contiene las discusiones y conclusiones resultantes de la investigación.

\section{Métodos}

\subsection{Diseño experimental.}

Se llevó a cabo un estudio descriptivo transversal con componente observacional para la medición de variables biomecánicas de variación angular en articulaciones de segmentos inferiores. El estudio se realizó en el Servicio de Amputados y Prótesis del Hospital Militar Central, Bogotá, Colombia. Las personas involucradas en el estudio fueron pacientes amputados transtibiales debido a trauma por minas antipersonas, todos hombres con amputación transtibial unilateral con edades que oscilan entre 29 y 40 años, con uso adecuado de prótesis por más de un año, con adaptación adecuada, Tabla 1. El tipo de prótesis es con sistema de suspensión por encaje interno y perno (liner y pin, en inglés), y pie de alta actividad en fibra de carbono.

\begin{tabular}{|c|c|c|c|c|}
\hline SUJETO & $\begin{array}{c}\text { LADO } \\
\text { AMPUTADo }\end{array}$ & $\begin{array}{c}\text { MASA } \\
(\mathbf{k g})\end{array}$ & $\begin{array}{c}\text { ESTATURA } \\
(\mathbf{c m})\end{array}$ & $\begin{array}{c}\text { EDAD } \\
\text { (años })\end{array}$ \\
\hline S1 & Derecho & 67 & 170 & 30 \\
\hline S2 & Izquierdo & 75 & 174 & 29 \\
\hline S3 & Izquierdo & 74 & 170 & 31 \\
\hline S4 & Izquierdo & 65 & 160 & 35 \\
\hline S5 & Izquierdo & 75 & 176 & 33 \\
\hline S6 & Izquierdo & 68 & 173 & 40 \\
\hline S7 & Derecho & 76 & 175 & 35 \\
\hline
\end{tabular}

Tabla 1. Relación de los sujetos participantes en la investigación, todos son amputados transtibiales y rehabilitados con uso adecuado de la prótesis. Fuente: elaboración propia 
El muestreo utilizado fue por conveniencia, ya que los sujetos son pacientes que consultan el Servicio de Amputados y Prótesis del Hospital Militar Central (en Bogotá, Colombia) durante un periodo de 3 meses, el número de participantes en el estudio fue 7 , correspondiente al 10\% de los pacientes nuevos por año que acuden al servicio.

El criterio de exclusión de los sujetos fue la presencia de alteraciones musculo esqueléticas o neurológicas en las otras extremidades, alteraciones sensoriales o cognitivas, lesiones en piel, alteraciones en marcha secundarias por dolor, uso de ayudas externas para la marcha, alteraciones articulares en otros segmentos corporales en miembros inferiores.

\subsection{Definición de las variables}

Se incluyeron variables de identificación del sujeto de estudio, variables antropométricas y variables a indagar como ubicación de la prótesis, ángulo de cadera, rodilla y tobillo de lado amputado y no amputado.

La medición de rangos articulares se realizó con el Sistema de Laboratorio de Adquisición de Datos, tipo No. LS900 de la empresa Biometrics Ltd ${ }^{\circ}$, que consta de goniómetros digitales de doble eje serie SG, un sistema de adquisición de datos (referencia DLK900), software de adquisición y tratamiento de señales DataLINK DLK900 versión software 5.0

\subsection{Mediciones}

Para realizar las mediciones, el sistema se instaló en el Hospital Militar Central (Bogotá, Colombia). La configuración del sistema se muestra en la Figura 1. El estudio fue desarrollado utilizando guías $2 \mathrm{D}$ para controlar la posición de los pies y de bipedestación durante la toma de muestras. Las guías colocan los pies del sujeto con puntos medios del talón a $150 \mathrm{~mm}$ de distancia y con un ángulo de progresión de $8^{\circ}$, teniendo en cuenta las condiciones propuestas por Lord y Smith [10]. Al inicio de cada sesión, se realizó un procedimiento de calibración utilizando la guía $2 \mathrm{D}$.

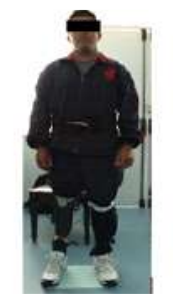

Figura 1. Configuración del sistema para realizar las mediciones de rangos articulares.

Fuente: elaboración propia.
Las diferentes ubicaciones angulares del encaje de la prótesis que se utilizaron en la presente investigación se muestran en la Figura 2. Se realizó flexión y extensión del encaje con respecto al pilón de la prótesis.

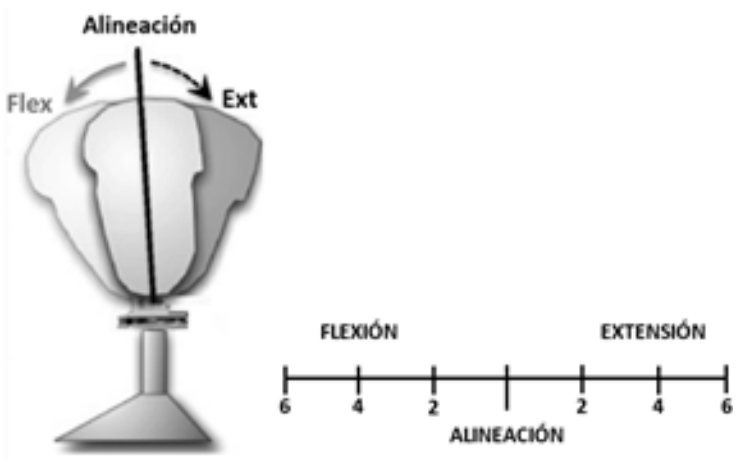

Figura 2. Posiciones del encaje de la prótesis utilizada en la investigación. Fuente: elaboración propia

Los valores de los datos obtenidos en las mediciones se almacenaron en una base de datos que se encuentra abierta al público. Esta página web será alimentada con los estudios que se realicen sobre el tema [11].

\section{Resultados}

En el análisis estadístico de los datos biomecánicos obtenidos en las mediciones [11], se observó que los datos de los sujetos en cada una de las medidas no son homogéneos, presentando diferencia tanto de medias como de varianzas. Se aplicó la prueba de Levene y la prueba $\mathrm{H}$ de Kruskal-Wallis, obteniendo un valor de significancia cercano a cero en ambas pruebas $(\mathrm{p}<0.05)$, luego se rechaza la hipótesis nula de homocedasticidad o igualdad de varianzas y la hipótesis nula de igualdad de promedios poblacionales, por tanto los datos de las mediciones de los sujetos entre sí son diferentes.

\begin{tabular}{|c|c|c|c|c|c|c|l|}
\hline VARIABLE & F6 & F4 & F2 & ALINEACIÓN & E2 & E4 & E6 \\
\hline CNA $\left(^{\circ}\right)$ & $-0,4$ & $-1,9$ & -1 & $-0,5$ & 2,4 & 1,8 & $-1,3$ \\
\hline RNA $\left(^{\circ}\right)$ & 1,1 & 2,2 & 1,4 & $-0,7$ & 2,1 & 3,3 & 3,8 \\
\hline TNA $\left(^{\circ}\right)$ & 3,6 & 4,9 & 2,2 & $-0,6$ & 3 & 2,6 & 6,3 \\
\hline CA $\left(^{\circ}\right)$ & $-2,7$ & $-0,3$ & 0 & 0,1 & $-2,2$ & $-0,7$ & 1,2 \\
\hline RA $\left(^{\circ}\right)$ & 7,2 & 8,2 & 3,8 & $-0,4$ & $-0,8$ & $-1,5$ & $-1,4$ \\
\hline TA $\left(^{\circ}\right)$ & 0,4 & 1,3 & 0,7 & $-0,0$ & $-0,7$ & $-1,1$ & $-0,3$ \\
\hline
\end{tabular}

Tabla 2. Valores estimados de la media de los rangos articulares. Fuente: elaboración propia. 
Se analizaron las variables biomecánicas en el estado inicial de la prótesis, es decir, en alineación, Tabla 2. Se hallaron diferencias significantes entre la pierna amputada y la no amputada. La mayor variación en las articulaciones se presente en las rodillas, la otra articulación con alta variabilidad es el tobillo de la pierna no amputada. La menor variación se da en el tobillo de la prótesis, confirmando su poca contribución a la estabilidad postural. Con respecto a la cadera, la contribución es mayor en el lado amputado.

La incidencia de la ubicación del encaje sobre los rangos articulares se realizó con el análisis de los datos. En los rangos articulares se determina que los sujetos ajustan las articulaciones cuando ocurre una alineación incorrecta, siendo el tobillo del lado no amputado el de mayor afectación cuando el encaje está en extensión, la rodilla de este lado también presenta alta afectación, este reporte concuerda con Isakov et al [12] y Xiaohong et al [13]; las articulaciones con mayor coeficiente de variación son cadera del lado amputado y tobillo del lado no amputado cuando el encaje se encuentra en extensión. Con el encaje en flexión la rodilla del lado amputado es donde mayor alteración se tiene seguido del tobillo del lado no amputado, pero las articulaciones con mayor índice de variación son las caderas. Así, en la pierna no amputada, las articulaciones de rodilla y tobillo están en flexión sin tener en cuenta la ubicación del encaje; el comportamiento de la articulación de la cadera varía dependiendo del ángulo del encaje. En la pierna amputada tanto la rodilla como el tobillo siguen los movimientos del encaje, esto es si el encaje se encuentra en flexión estas dos articulaciones también y si se encuentra en extensión las articulaciones igualmente, se debe anotar que el movimiento del tobillo es mínimo, menos de un grado; en el caso de la cadera, su posición angular se altera según la posición del ángulo del encaje. Las variaciones de los ángulos de las articulaciones se deben a los mecanismos de control de estabilidad que realizan los pacientes para no perder el equilibrio y caerse, entonces el tobillo del lado no amputado es el que más aporte hace al mecanismo de compensación cuando el encaje está en extensión, y en flexión es la rodilla del lado no amputado.

\section{Conclusiones}

Mediante el uso de equipos electrónicos de medición de ángulos de articulaciones, se pudo obtener importante información sobre el comportamiento de estas variables biomecánicas en la alineación estática de prótesis transtibiales. Además se consolidó una base de datos de la alineación estática que incluye variables cinéticas [11].

Durante el estudio y recolección de datos se estableció un protocolo de medición que permitió controlar la posición del individuo, el tiempo de duración de la medición, la sincronización de los equipos de medición, así como establecer la ubicación correcta de los dispositivos en la estructura anatómica del sujeto y realizar cambios controlados en la ubicación angular del encaje para asegurar el ángulo presentado por éste. Se requirió el uso de guías $2 \mathrm{D}$ en el piso para posicionar los pies de los pacientes.

Al comparar los valores de las mediciones entre los miembros inferiores, es evidente la asimetría entre la extremidad no amputada y la amputada. Como los usuarios de prótesis transtibial carecen de un tobillo anatómico y por tanto de todas las estructuras sensoriomotoras que involucran este segmento anatómico, son incapaces de mantener la estabilidad postural con una estrategia de tobillo en el lado protésico, de allí que la variabilidad de este rango articular sea insignificante. Para mantener la estabilidad postural deben compensar utilizando las estructuras restantes, una estrategia postural modificada es el uso de la rodilla.

Los rangos articulares de miembros inferiores se ven afectados por la ubicación de la prótesis, esto concuerda con Isakov et al [12], [13], Xiaohong et al [14] y Engsberg et al [15]. La estabilidad postural se ve disminuida en personas con amputación transtibial como resultado de cambios biomecánicos y neurofisiológicos, lo cual conduce a mecanismos de adaptación y compensación -[1618], luego al tener el encaje en extensión el tobillo del lado no amputado es el que más aporte hace al mecanismo de compensación para no perder estabilidad, y en flexión es la rodilla del lado no amputado, estos resultados ratifican la conclusión de Buckley et al [19], ya que ellos muestran la importancia del tobillo para mantener el equilibrio en situaciones que involucran los movimientos del cuerpo en el plano sagital.

\section{Referencias}

[1] M. Leblanc, "Give Hope - Give a Hand - The LN4 Prosthetic Hand”, 2008. [En línea]. Disponible enhttp://www.stanford.edu/class/engr 110/20 11/LeBlanc-03a.pdf.

[2] DANE y CENSO 2005, "Discapacidad Personas con limitaciones permanentes”, 2006. [En línea]. Disponible en: 
http//www.dane.gov.co/files/censo2005/discapa cidad.pdf.

[3] Dirección Contra Minas, "Víctimas de Minas Antipersonal", 2014. [En línea]. Disponible e $\mathrm{n}$

http://www.accioncontraminas.gov.co/estad is ticas / Paginas / victimas-minasantipersonal.aspx.

[4] M. A. González, O. Cohí y F. Salinas, "Amputación de extremidad inferior y discapacidad: Prótesis y rehabilitación". Barcelona: Masson, 2005.

[5] L. A. Luengas C., M. A. Gutierrez y E. Camargo, "Alineación de prótesis y parámetros biomecánicos de pacientes amputados transtibiales". Bogota: UD Editorial, 2017.

[6] M. S. Pinzur, W. Cox, J. Kaiser, T. Morris, A. Patwardhan y L. Vrbos, "The effect of prosthetic alignment on relative limb loading in persons with trans-tibial amputation: a preliminary report", J. Rehabil. Res. Dev., vol. 32, no. 4, 1995, pp. 373-378.

[7] S. Blumentritt, T. Schmalz, R. Jarasch y M. Schneider, "Effects of sagittal plane prosthetic alignment on standing trans-tibial amputee knee loads", Prosthet. Orthot. Int., vol. 23, no. 3, 1999 , p p. $231-238$. D O I : https://doi.org/10.3109/03093649909071639.

[8] D. Boone, T. Kobayashi, T. G. Chou, A. K. Arabian, K. L. Coleman, M. S. Orendurff y M. Zhang, "Influence of malalignment on socket reaction moments during gait in amputees with transtibial prostheses", Gait Posture, vol. 37, no. 4,2012 , p p. $620-626$. D O I : https://doi.org/10.1016/j.gaitpost.2012.10.00 2.

[9] M. J. Nederhand, E. H. F. Van Asseldonk, H. van der Kooij y H. S. Rietman, "Dynamic Balance Control (DBC) in lower leg amputee subjects; contribution of the regulatory activity of the prosthesis side", Clin. Biomech. (Bristol, Avon), vol. 27, no. 1, 2012 , pp. 40-50. DOI: https://doi.org/10.1016/j.clinbiomech.2011.0 7.008 .

[10] M. Lord y D. M. Smith, "Foot loading in amputee stance", Prosthet. Orthot. Int., vol. 8, no. 3, 1984, p p. $159-64$. D O I :

https://doi.org/10.3109/030936484091460
79 .

[11] L. Luengas y J. López, "Alineamtranstib”, 2017. [En línea]. Dis ponible e n : https://alineamtranstib.wixsite.com/alineamtr anstib.

[12] E. Isakov, J. Mizrahi, H. Ring, Z. Susak y N. Hakim, "Standing sway and weight-bearing distribution in people with below-knee amputations", Arch. Phys. Med. Rehabil., vol. 73, no. 2, 1992, pp. 174-178.

[13] E. Isakov, J. Mizrahi, Z. Susak, I. Ona y N. Hakim, "Influence of prosthesis alignment on the standing balance of below-knee amputees", Clin. Biomech., vol. 9, no. 4, 1994, pp. 258-262. DOI: https://doi.org/10.1016/02680033(94)90008-6.

[14] J. Xiaohong, L. Xiaobing, D. Peng y Z. Ming, "The Influence of Dynamic Trans-tibial Prosthetic Alignment on Standing Plantar Foot Pressure", Conf. Proc. IEEE Eng. Med. Biol. Soc., vol. 7, 2005, pp. 6916-6918. DOI: https://doi.org/10.1109/IEMBS.2005.161609 6.

[15] J. R. Engsberg, T. L. Allinger y J. A. Harder, "Standing pressure distribution for normal and below-knee amputee children", Prosthet. Orthot. Int., vol. 13,no. 3, 1989, pp. 152-155. D O I .

https://doi.org/10.3109/03093648909079425.

[16] D. Kozáková, Z. Svoboda, M. Janura, M. Elfmark y I. Nedvědová, "Assessment of Postural Stability in Patients With a Transtibial Amputation With Various Times of Prosthesis Use", Acta Univ. Palacki. Olomuc, vol. 39, no. 3, 2009, pp. 51-59.

[17] Á. A. Mayer, "The body awareness and the standing stability of amputees", tesis Ph.D., Semmelweis University, Budapest, 2011.

[18] B. Paráková, M. Míková y M. Janura, "The influence of prostheses and prosthetic foot alignment on postural behavior in transtibial amputees", Acta Univ. Palacki. Olomuc. Gymnica, vol. 37, no. 4, 2007, pp. 37-44.

[19] J. Buckley, D. O'Driscoll y S. Bennett, "Postural sway and active balance performance in highly active lower-limb amputees", Am. J. Phys., vol. 81 , no. 1, 2002, pp. 13-20. DOI: http://doi.org/10.1097/00002060200201000-00004. 